\title{
Playing with Fire? A Safe and Effective Deactivation of Raney Cobalt using Aqueous Sodium Nitrate
}

John Jin Lim ${ }^{\star \dagger}$, Frank Dixon Jr. ${ }^{\S}$, David C. Leitch ${ }^{1 \dagger}$, John Kowalski ${ }^{\dagger}$, Mark Nilson ${ }^{\dagger}$, Charles Goss ${ }^{\ddagger}$, Roy

Flanagan $^{2}$, Sean Hayes ${ }^{3}$, Michael J. Murphy ${ }^{3}$.

†Chemical Development, API Chemistry, GlaxoSmithKline, Upper Providence, PA, 19426, USA.

${ }^{1}$ Department of Chemistry, University of Victoria, Victoria, BC, V8P 5C2, Canada.

${ }^{\ddagger}$ Chemical Development, Product and Process Engineering, GlaxoSmithKline, Upper Providence, PA, 19426, USA.

SProduct Development \& Supply, Process Safety, GlaxoSmithKline, Upper Providence, PA, 19426, USA. ${ }^{2}$ Product Development \& Supply, Process Safety, GlaxoSmithKline, Zebulon, NC, 27597. USA.

${ }^{3}$ NPI Technical, Pharma Supply Chain, GlaxoSmithKline, Cork, T12 P6PT, Ireland.

\section{Supporting Information}

Figure S1: Equipment set-up (probes, dosing pump, and mass flow meter not shown)

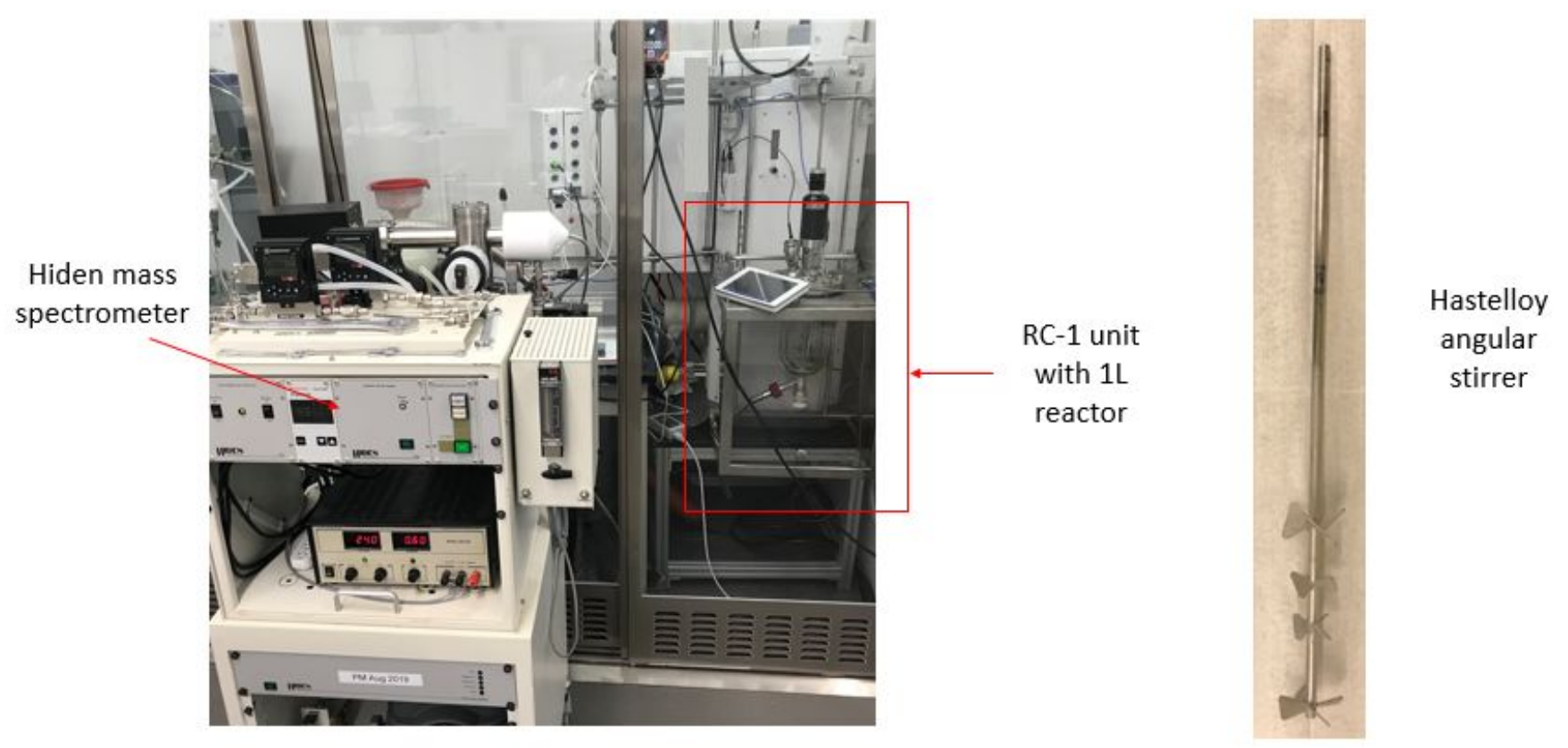


Table S1: Calorimetry data for sodium nitrate and bleach quenches of Raney cobalt

\begin{tabular}{|c|c|c|c|c|c|c|c|c|c|c|c|}
\hline $\begin{array}{l}\text { Raney } \\
\text { cobalt } \\
\text { charged } \\
\text { (g) }\end{array}$ & $\begin{array}{l}\text { Quenching } \\
\text { solution }\end{array}$ & $\begin{array}{c}\text { Heat } \\
\text { capacity } \\
\left(k J / \mathrm{kg}^{\circ} \mathrm{K}\right)\end{array}$ & $\begin{array}{c}\text { Dose } \\
1 \text { heat } \\
\text { output } \\
\text { (kJ) }\end{array}$ & $\begin{array}{c}\text { Dose } \\
2 \text { heat } \\
\text { output } \\
(k J)\end{array}$ & $\begin{array}{c}\text { Dose } \\
3 \text { heat } \\
\text { output } \\
(k J)\end{array}$ & $\begin{array}{c}\text { Total } \\
\text { heat } \\
\text { output } \\
\text { (kJ/kg } \\
\text { catalyst) }\end{array}$ & $\begin{array}{c}\text { Dose } 1 \\
\text { adiabatic } \\
\text { temperature } \\
\text { rise }\left({ }^{\circ} \mathrm{C}\right)\end{array}$ & $\begin{array}{c}\text { Dose } 2 \\
\text { adiabatic } \\
\text { temperature } \\
\text { rise }\left({ }^{\circ} \mathrm{C}\right)\end{array}$ & $\begin{array}{c}\text { Dose } 3 \\
\text { adiabatic } \\
\text { temperature } \\
\text { rise }\left({ }^{\circ} \mathrm{C}\right)\end{array}$ & $\begin{array}{c}\text { Total } \\
\text { adiabatic } \\
\text { temperature } \\
\text { rise }\left({ }^{\circ} \mathrm{C}\right)\end{array}$ & $\begin{array}{c}\mathrm{O}_{2} \\
\text { released } \\
(\mathrm{L} / \mathrm{kg} \\
\text { catalyst })\end{array}$ \\
\hline 35 & $5 \%$ bleach & 3.66 & 70.7 & 56.3 & 5.3 & 3780 & 32.9 & 18.7 & 1.6 & 53 & 91 \\
\hline 35 & $\begin{array}{c}5 \% \\
\text { sodium } \\
\text { nitrate }\end{array}$ & 3.80 & 33.1 & 2.8 & & 1024 & 12.0 & $<1.0$ & & 12 & none \\
\hline
\end{tabular}

\section{Details of CHETAH calculations}

CHETAH is a predictive software tool licensed by ASTM International and is commonly used by process safety scientists to predict the expected heats of reactions. The calculation was performed by assuming $\sim 51 \% \mathrm{H}_{2}$ absorption on the cobalt surface (ie. the cobalt hydrides which are reduced by the quench methods), which was determined by retrospective computation of activated Co required to generate the experimentally observed amount of oxygen, using the balanced bleach quenching equations. This degree of hydrogen adsorption is well supported by the literature ${ }^{1-3}$. For the bleach quench, we propose the following reaction equation:

$3 \mathrm{NaOCl}+2 \mathrm{Co}-\mathrm{H} \rightarrow \mathrm{O}_{2}+\mathrm{H}_{2} \mathrm{O}+3 \mathrm{NaCl}+2 \mathrm{Co}$

A $1 \mathrm{~kg}$ sample of Raney cobalt (which is $86 \%$ wt cobalt according to the supplier spec sheet) contains $14.6 \mathrm{~mol}$ of cobalt $=1 \mathrm{~kg} \times 0.86 \times 58.9 \mathrm{~g} / \mathrm{mol}$ (MW of cobalt).

$14.6 \mathrm{~mol}$ of cobalt with $51 \% \mathrm{H}_{2}$ absorption $=7.45 \mathrm{~mol}$ of $\mathrm{Co}-\mathrm{H}$.

The ratio of $\mathrm{NaOCl}: \mathrm{Co}-\mathrm{H}=3: 2$, so $7.45 \mathrm{~mol} \times 1.5=11.18 \mathrm{~mol} \mathrm{NaOCl}$ to quench $1 \mathrm{~kg}$ catalyst. 
Because the bond energy values of $\mathrm{Co}-\mathrm{H}$ are not available in $\mathrm{CHETAH}$, we substituted the 2 molecules of Co-H with 1 molecule of $\mathrm{H}_{2}$ in the balanced equation to perform the CHETAH calculation. We thus expect some error due to the differences in bond dissociation energies (BDE's) reported in the literature for $\mathrm{Co}-\mathrm{H}$ and $\mathrm{H}_{2}$, respectively ${ }^{4,5}$. CHETAH calculations are ideally performed using energy values in the gas phase. For this reason, substitution of $\mathrm{Na}^{+}$for $\mathrm{H}^{+}$is a common approach used by practitioners; therefore, we substituted $\mathrm{HOCl}$ for sodium hypochlorite.

\section{$\underline{\text { CHETAH calculation for the bleach quench }}$}

$3 \mathrm{HOCl}+\mathrm{H}_{2}($ from $2 \mathrm{Co}-\mathrm{H}) \rightarrow \mathrm{O}_{2}+\mathrm{H}_{2} \mathrm{O}+3 \mathrm{HCl}(-70.6 \mathrm{kcal} / \mathrm{mol})$

Therefore,

$-70.6 \mathrm{kcal} / \mathrm{mol}(\mathrm{HOCl}) \times 11.18 \mathrm{~mol} \mathrm{HOCl} / \mathrm{kg}$ catalyst $=-833.1 \mathrm{kcal} / \mathrm{kg}$ catalyst $=-3485.7 \mathrm{~kJ} / \mathrm{kg}$ catalyst, which is close to the observed value in Table $\mathrm{S} 1$.

The calculation for the sodium nitrate quench is performed in the analogous fashion. For the sodium nitrate quench, we propose the following reaction equation:

$$
2 \mathrm{NaNO}_{3}+10 \mathrm{Co}-\mathrm{H} \rightarrow \mathrm{N}_{2}+4 \mathrm{H}_{2} \mathrm{O}+2 \mathrm{NaOH}+10 \mathrm{Co}
$$

The ratio of $\mathrm{NaNO}_{3}: \mathrm{Co}-\mathrm{H}=2: 10$, so $7.45 \mathrm{~mol}$ of $\mathrm{Co}-\mathrm{H} / 5=1.49 \mathrm{~mol} \mathrm{NaNO}$ to quench $1 \mathrm{~kg}$ catalyst

Because the bond energy values of $\mathrm{Co}-\mathrm{H}$ are not available in CHETAH, we substituted the 10 molecules of $\mathrm{Co}-\mathrm{H}$ with 5 molecule of $\mathrm{H}_{2}$ in order to perform the calculation. We thus expect some error due to the differences in bond dissociation energies (BDE's) reported in the literature Co-H and $\mathrm{H}_{2}$, respectively ${ }^{4,5}$. In addition, because the calculations are performed using gas phase bond energy values, we substituted nitric acid for sodium nitrate.

\section{$\underline{\text { CHETAH calculation for sodium nitrate quench }}$}

$2 \mathrm{HNO}_{3}+5 \mathrm{H}_{2}($ from $10 \mathrm{Co}-\mathrm{H}) \rightarrow \mathrm{N}_{2}+6 \mathrm{H}_{2} \mathrm{O} \quad(-158 \mathrm{kcal} / \mathrm{mol})$ 
Therefore,

$-158 \mathrm{kcal} / \mathrm{mol}\left(\mathrm{HNO}_{3}\right) \times 1.49 \mathrm{~mol} \mathrm{HNO}_{3} / \mathrm{kg}$ catalyst $=-235.3 \mathrm{kcal} / \mathrm{kg}$ catalyst $=-984.7 \mathrm{~kJ} / \mathrm{kg}$ catalyst, which is close to the observed value in Table S1. Raw data as follows:

GlaxosmithKline Research \& Dev Ltd

Reaction Thermochemistry Report

Session/Report Title: Raney Co Bleach Quench

\begin{tabular}{|c|c|c|c|c|c|}
\hline Reaction: 3 & $\mathrm{HO}(\mathrm{g})+\mathrm{H}_{2}$ & ref) $\ldots 3 c$ & (g) $+\mathrm{H} 2 \mathrm{O}$ & (g) +02 (ref) & \\
\hline $\begin{array}{c}\text { Temp } \\
{ }^{\circ} \mathrm{K}\end{array}$ & $\begin{array}{l}\text { delHrxn } \\
\mathrm{kcal} / \mathrm{mol}\end{array}$ & $\begin{array}{l}\text { delGrxn } \\
\mathrm{kcal} / \mathrm{mol}\end{array}$ & $\log K$ & $\begin{array}{c}\text { delCp } \\
\mathrm{cal} /(\mathrm{mol}-\mathrm{K})\end{array}$ & $\begin{array}{c}\text { delSrxn } \\
\mathrm{cal} /(\mathrm{mol}-\mathrm{K})\end{array}$ \\
\hline 298.15 & -70.580 & -78.698 & 57.687 & 2.308 & 27.229 \\
\hline 348.15 & -70.487 & -80.068 & 50.261 & 1.401 & 27.518 \\
\hline 400.00 & -70.437 & -81.499 & 44.528 & 0.577 & 27.654 \\
\hline
\end{tabular}

\section{Reactants:}

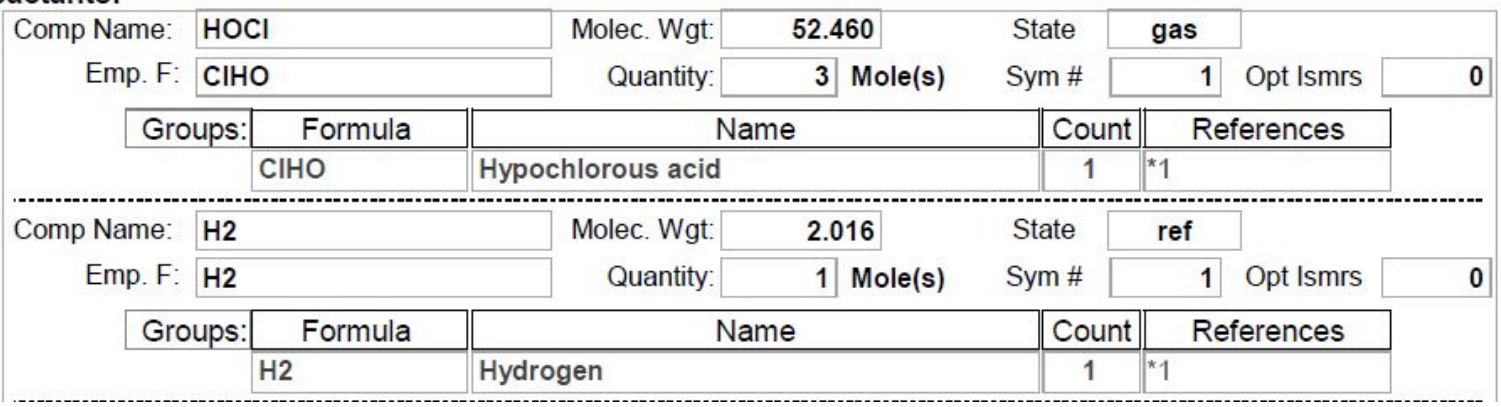




\begin{tabular}{|c|c|c|c|c|c|c|c|c|c|}
\hline \multicolumn{10}{|c|}{ Products: } \\
\hline \multirow{2}{*}{$\begin{array}{r}\text { Comp Name: } \\
\text { Emp. F: }\end{array}$} & \multirow{2}{*}{\multicolumn{2}{|c|}{$\begin{array}{l}\mathrm{HCl} \\
\mathrm{CIH}\end{array}$}} & \multirow{2}{*}{$\begin{array}{r}\text { Molec. Wgt: } \\
\text { Quantity: }\end{array}$} & \multicolumn{2}{|c|}{36.461} & \multirow{2}{*}{$\begin{array}{l}\text { State } \\
\text { Sym \# }\end{array}$} & \multirow{2}{*}{$\begin{array}{r}\text { gas } \\
1\end{array}$} & \multirow[b]{2}{*}{ Opt Ismrs } & \multirow[b]{2}{*}{0} \\
\hline & & & & 3 & Mole(s) & & & & \\
\hline \multirow{2}{*}{\multicolumn{2}{|c|}{ Groups: }} & Formula & \multicolumn{3}{|c|}{ Name } & Count & \multicolumn{2}{|c|}{ References } & \\
\hline & & $\mathrm{CIH}$ & \multicolumn{3}{|l|}{ Hydrogenchloride } & 1 & \multicolumn{2}{|c|}{ *1 } & \\
\hline \multirow{2}{*}{$\begin{array}{r}\text { Comp Name: } \\
\text { Emp. F: }\end{array}$} & \multicolumn{2}{|c|}{ water } & Molec. Wgt: & \multicolumn{2}{|c|}{18.015} & State & gas & & \\
\hline & \multicolumn{2}{|c|}{$\mathrm{H} 2 \mathrm{O}$} & Quantity: & 1 & Mole(s) & Sym \# & 2 & Opt Ismrs & 0 \\
\hline \multirow{2}{*}{\multicolumn{2}{|c|}{ Groups: }} & Formula & \multicolumn{3}{|c|}{ Name } & Count & \multicolumn{2}{|c|}{ References } & \\
\hline & & $\mathrm{H} 2 \mathrm{O}$ & \multicolumn{3}{|l|}{ Water Ideal Gas } & 1 & \multicolumn{2}{|c|}{ *1 } & \\
\hline \multirow{2}{*}{$\begin{array}{r}\text { Comp Name: } \\
\text { Emp. F: }\end{array}$} & \multicolumn{2}{|l|}{$\mathrm{O} 2$} & Molec. Wgt: & \multicolumn{2}{|c|}{31.999} & State & ref & \multirow[b]{2}{*}{ Opt Ismrs } & \\
\hline & \multicolumn{2}{|l|}{$\mathrm{O} 2$} & Quantity: & 1 & Mole(s) & Sym \# & 1 & & 0 \\
\hline \multirow{2}{*}{\multicolumn{2}{|c|}{ Groups: }} & Formula & \multicolumn{3}{|c|}{ Name } & Count & \multicolumn{2}{|c|}{ References } & \\
\hline & & $\mathrm{O} 2$ & \multicolumn{3}{|l|}{ Oxygen } & 1 & \multicolumn{2}{|c|}{ *1 } & \\
\hline
\end{tabular}

\section{Literature References:}

References for: $\mathrm{HOCl}$

*1 Chase,M.W., Davies,C.A., Downey,J.R., Frurip,D.J., McDonald,R.A. and Syverud,A.N., "JANAF Thermochemical Tables", third ed. J. Phys. Chem. Ref. Data, vol. 14, supp. 1, 1985.

\section{References for: $\mathrm{H} 2$}

*1 Robie,R.A., Hemingway,B.S., and Fisher,J.R., Thermodynamic Properties Of Minerals and Related Substances at $298.15 \mathrm{~K}$ and 1 Bar Pressure and At Higher Temperatures, U.S Geol.Survey Bull. 1452, (1979).

\section{References for: $\mathrm{HCl}$}

*1 (a). Stull,D.R., et al, JANAF Thermochemical Data, 1963-Date. (b). Stull,D.R., and Sinke,G.C., "Thermodynamic Properties Of The Elements," ACS Advances In Chemistry Series No. 18, American Chemical Society, Washington, D.C., 1956.

\section{References for: water}

*1 Chase,M.W., Davies,C.A., Downey,J.R., Frurip,D.J., McDonald,R.A. and Syverud,A.N., "JANAF Thermochemical Tables", third ed., J. Phys. Chem. Ref. Data, vol. 14, supp. 1, 1985.

\section{References for: $\mathrm{O} 2$}

*1 Robie,R.A., Hemingway,B.S., and Fisher,J.R., Thermodynamic Properties Of Minerals and Related Substances at 298.15 K and 1 Bar Pressure and At Higher Temperatures, U.S Geol.Survey Bull. 1452, (1979). 
Session/Report Title: Raney Co Nitrate Quench

\begin{tabular}{|c|c|c|c|c|c|}
\hline \multicolumn{6}{|l|}{ Reaction: } \\
\hline $\begin{array}{c}\text { Temp } \\
{ }^{\circ} \mathrm{K}\end{array}$ & $\begin{array}{l}\text { delHrxn } \\
\text { kcal/mol }\end{array}$ & $\begin{array}{l}\text { delGrxn } \\
\mathrm{kcal} / \mathrm{mol}\end{array}$ & $\log K$ & $\begin{array}{c}\text { delCp } \\
\mathrm{cal} /(\mathrm{mol}-\mathrm{K})\end{array}$ & $\begin{array}{c}\text { delSrxn } \\
\mathrm{cal} /(\mathrm{mol}-\mathrm{K})\end{array}$ \\
\hline 298.15 & -157.915 & & & 66.749 & \\
\hline 348.15 & -154.615 & & & 65.153 & \\
\hline 400.00 & -151.281 & & & 63.567 & \\
\hline
\end{tabular}

(Full Thermodynamic Table cannot be done because of missing Entropy Data)

\section{Reactants:}

\begin{tabular}{|c|c|c|c|c|c|c|c|c|c|}
\hline \multirow{2}{*}{$\begin{array}{r}\text { Comp Name: } \\
\text { Emp. F: }\end{array}$} & \multirow{2}{*}{\multicolumn{2}{|c|}{$\begin{array}{l}\text { HNO3 } \\
\text { HNO3 }\end{array}$}} & \multirow{2}{*}{$\begin{array}{r}\text { Molec. Wgt: } \\
\text { Quantity: }\end{array}$} & \multicolumn{2}{|c|}{63.013} & \multirow{2}{*}{$\begin{array}{l}\text { State } \\
\text { Sym \# }\end{array}$} & \multirow{2}{*}{$\frac{a q}{1}$} & \multirow[b]{2}{*}{ Opt Ismrs } & \multirow[b]{2}{*}{0} \\
\hline & & & & 1 & Mole(s) & & & & \\
\hline $\mathrm{Gr}$ & ups:| & Formula & \multicolumn{3}{|c|}{ Name } & Count & \multicolumn{2}{|c|}{ References } & \\
\hline & & HNO3 & \multicolumn{3}{|l|}{ HNO3 ai } & 1 & \multicolumn{2}{|l|}{ *1 } & \\
\hline
\end{tabular}

\begin{tabular}{|c|c|c|c|c|c|c|c|c|c|c|}
\hline \multirow{2}{*}{$\begin{array}{r}\text { Comp Name: } \\
\text { Emp. F: }\end{array}$} & \multicolumn{3}{|l|}{$\mathrm{H} 2$} & \multirow{2}{*}{$\begin{array}{r}\text { Molec. Wgt: } \\
\text { Quantity: }\end{array}$} & \multicolumn{2}{|c|}{2.016} & \multirow{2}{*}{$\begin{array}{l}\text { State } \\
\text { Sym \# }\end{array}$} & \multirow{2}{*}{ aq } & \multirow[b]{2}{*}{ Opt Ismrs } & \multirow[b]{2}{*}{0} \\
\hline & $\mathrm{H} 2$ & & & & 2.5 & Mole(s) & & & & \\
\hline \multirow{2}{*}{\multicolumn{2}{|c|}{ Groups: }} & Formula & \multicolumn{4}{|c|}{ Name } & Count & \multicolumn{2}{|c|}{ References } & \\
\hline & & $\mathrm{H} 2$ & $\mathrm{H} 2 \mathrm{ao}$ & & & & 1 & \multicolumn{2}{|c|}{ *1 } & \\
\hline
\end{tabular}

\section{Products:}

\begin{tabular}{|c|c|c|c|c|c|c|c|c|c|}
\hline \multirow{2}{*}{$\begin{array}{r}\text { Comp Name: } \\
\text { Emp. F: }\end{array}$} & \multirow{2}{*}{\multicolumn{2}{|c|}{$\begin{array}{l}\text { N2 } \\
\text { N2 }\end{array}$}} & \multirow{2}{*}{$\begin{array}{r}\text { Molec. Wgt: } \\
\text { Quantity: }\end{array}$} & \multicolumn{2}{|c|}{28.013} & State & ref & \multirow[b]{2}{*}{ Opt Ismrs } & \multirow[b]{2}{*}{0} \\
\hline & & & & 0.5 & Mole(s) & Sym \# & 1 & & \\
\hline \multirow{2}{*}{\multicolumn{2}{|c|}{ Groups: }} & Formula & \multicolumn{3}{|c|}{ Name } & Count & \multicolumn{2}{|c|}{ References } & \\
\hline & & N2 & Nitrogen & & & 1 & & & \\
\hline \multirow{2}{*}{$\begin{array}{r}\text { Comp Name: } \\
\text { Emp. F: }\end{array}$} & \multicolumn{2}{|c|}{ water } & Molec. Wgt: & \multicolumn{2}{|c|}{18.015} & State & $\mathrm{aq}$ & & \\
\hline & \multicolumn{2}{|c|}{$\mathrm{H} 2 \mathrm{O}$} & Quantity: & 3 & Mole(s) & Sym \# & 1 & Opt Ismrs & 0 \\
\hline \multirow{2}{*}{\multicolumn{2}{|c|}{ Groups: }} & \begin{tabular}{|l|} 
Formula \\
\end{tabular} & \multicolumn{3}{|c|}{ Name } & Count & \multicolumn{2}{|c|}{ References } & \\
\hline & & $\mathrm{H} 2 \mathrm{O}$ & \multicolumn{3}{|l|}{ Water Liquid } & 1 & \multicolumn{2}{|l|}{ *1 } & \\
\hline
\end{tabular}

\section{Literature References:}

References for: $\mathrm{HNO} 3$

*1 D.D. Wagman, W.H. Evans, V.B. Parker, R.H. Schumm, I. Halow, S.M. Bailey, K.L. Churney, and R. L. Nuttall, The NBS Tables of Chemical and Thermodynamic Properties, Journal of Physical Chemical Reference Data, Vol. 11, 1982, Supplement No. 2.

\section{References for: $\mathrm{H} 2$}

*1 D.D. Wagman, W.H. Evans, V.B. Parker, R.H. Schumm, I. Halow, S.M. Bailey, K.L. Churney, and R. L. Nuttall, The NBS Tables of Chemical and Thermodynamic Properties, Journal of Physical Chemical Reference Data, Vol. 11, 1982, Supplement No. 2.

\section{References for: N2}

*1 Robie,R.A., Hemingway,B.S., and Fisher,J.R., Thermodynamic Properties Of Minerals and Related Substances at $298.15 \mathrm{~K}$ and 1 Bar Pressure and At Higher Temperatures, U.S Geol.Survey Bull. 1452, (1979).

References for: water

*1 $\quad$ Chase, M.W., Jr., NIST-JANAF Themochemical Tables, Fourth Edition, J.Phys. Chem. Ref. Data, Monograph 9,1998,1-1951 


\section{REFERENCES}

1. Smith, H.; Chadwell, A.; Kinlis, S. The Role of Hydrogen in Raney Nickel Catalyst. J. Phys. Chem. 1955, 59, 820-822.

2. Kokes, R.J.; Emmett, P. H. The Role of Hydrogen in Raney Nickel Catalysts. J. Am. Chem. Soc. 1959, 81, 5032-5037.

3. Comprehensive Organic Name Reactions and Reagents https://doi.org/10.1002/9780470638859.conrr520

4. Georgiadis, R.; Fisher, E.R.; Armentrout, P.B. Neutral and Ionic Metal-Hydrogen and MetalCarbon Bond Energies: Reactions of $\mathrm{Co}^{+}, \mathrm{Ni}^{+}$, and $\mathrm{Cu}+$ with Ethane, Propane, Methylpropane, and Dimethylpropane. J. Am. Chem. Soc. 1989, 111, 4251-4262.

5. Wagman, D.D.; Evans, W.H.; Parker, V.B.; Schumm R.H.; Halow, I.; Bailey, S.M.; Churney, K.L.; Nuttall, R.L. The NBS Tables of Chemical and Thermodynamic Properties, Journal of Physical Chemical Reference Data. 1982, 11, Supplement No. 2. 